\title{
Natural Language Processing Empowered Mobile Computing
}

\author{
Tianyong Hao $\mathbb{D}$, ${ }^{1}$ Raymond Wong $\left(\mathbb{D},{ }^{2}\right.$ Zhe He $\mathbb{D},{ }^{3}$ Haoran Xie, \\ Tak-Lam Wong ${ }^{(D)}{ }^{5}$ and Fu Lee Wang $\mathbb{D}^{6}$ \\ ${ }^{1}$ South China Normal University, Guangzhou, China \\ ${ }^{2}$ University of New South Wales, Sydney, Australia \\ ${ }^{3}$ Florida State University, Tallahassee, USA \\ ${ }^{4}$ The Education University of Hong Kong, New Territories, Hong Kong \\ ${ }^{5}$ Douglas College, British Columbia, Canada \\ ${ }^{6}$ The Open University of Hong Kong, Hong Kong \\ Correspondence should be addressed to Tianyong Hao; haoty@gdufs.edu.cn
}

Received 13 September 2018; Accepted 13 September 2018; Published 14 October 2018

Copyright (c) 2018 Tianyong Hao et al. This is an open access article distributed under the Creative Commons Attribution License, which permits unrestricted use, distribution, and reproduction in any medium, provided the original work is properly cited.

With the rapid growth of mobile device usage, more and more mobile contents offer a great opportunity for mining useful information. However, these contents mostly exist in free-text format in web pages, news feeds, SMS, and Twitter/WeChat messages, posing a significant challenge for information extraction. Natural language processing (NLP) is an important field of computer science, artificial intelligence, and computational linguistics. It is concerned with the interactions between computers and human (natural) language. NLP aims to enable a computer program to process and understand unstructured texts. In the mobile environment, NLP can make apps smarter by automatically analyzing the content, understanding its semantics, and taking appropriate actions on behalf of their users. The state-of-the-art NLP techniques have proven to be useful in dealing with the information overload problem in the mobile environment, e.g., news aggregation and summarization, question answering, information extraction and retrieval, semantic understanding, and personalization.

Applying NLP techniques to the content on mobile devices has recently gained attention in both academia and industry. Efforts have been made to develop various NLP applications for mobile platforms, such as Siri, which can retrieve information about places or events, and Google Now, which can extract context-sensitive information. However, it still lacks deep text processing capabilities, such as abstraction, summarization, and semantic understanding to accomplish complex and information-intensive tasks. Beyond the common applications, in the fast growing areas, such as healthcare, critical electronic health record data are accessible almost anywhere and near instantly even in ICU. Patient notes can be retrieved with a few taps on a screen. Sophisticated NLP techniques are required for analyzing the huge volume of data timely for making critical decisions.

In this special issue, articles regarding the use of technologies, methodologies, and applications for natural language processing and mobile computing were submitted and peerreviewed. The special issue shows a diversity of new developments in these areas. We accepted 7 high-quality original research articles and 1 review article.

The paper "A Bibliometric Review of Natural Language Processing Empowered Mobile Computing" introduced a systematical investigation and analysis on the scholarly publications on the topic of natural language processing empowered mobile computing in the last ten years. The authors applied a number of analytical techniques including descriptive statistics, geographic visualization, social network analysis, Latent Dirichlet Allocation, and affinity propagation clustering, to discover the status of research efforts and the trend of the topic. The paper can potentially help researchers understand research hot spots, collaboration patterns, and scholarly resource distribution as well as trace new scientific development in the field. 
The paper "The Current Status and a New Approach for Chinese Doctors to Obtain Medical Knowledge Using Social Media: A Study of WeChat" provided a quantitatively exploration of the approaches to acquiring medical knowledge using social media such as WeChat. Focusing on WeChat, the most widely used mobile social media in China, the authors designed, distributed, collected, and analyzed a selfadministered questionnaire utilizing an online survey tool. The paper reported the most desirable mode for acquiring professional medical knowledge through WeChat from data analysis. The paper advocates both academia and industry to pay more attention to social media such as WeChat for its increasingly important role in acquiring medical knowledge and continuing education for Chinese doctors.

The paper "Recognizing Continuous and Discontinuous Adverse Drug Reaction Mentions from Social Media Using LSTM-CRF" presented an important use of social media in medicine, particularly concerning the treatment experiences of patients using mobile devices with valuable adverse drug reaction information (ADR). The authors proposed a deep neural network LSTM-CRF by combining LSTM neural networks with CRFs to recognize ADR mentions from social media in medicine. They investigated the effects of three factors including representation for continuous and discontinuous ADR mentions, subject of posts, and external knowledge bases. The authors believe that the paper is the first attempt to use deep neural networks for mining continuous and discontinuous ADRs from social media.

The paper "A Mobile-Based Question-Answering and Early Warning System for Assisting Diabetes Management” showed that developing a convenient device on chronic disease management and monitoring is increasingly important. Focusing on type 2 diabetes, the authors developed a mobile-based diabetes question-answering and early warning system named Dia-AID, which assisted diabetes patients or people with a high risk of diabetes. The system presented a large multilanguage repository of frequently asked diabetes questions, a multimode fusion Q\&A framework, and a health data management module. The system is expected to assist diabetes patients or people at a high risk of diabetes in providing diabetes information and monitoring their health status through diabetes question answering, risk assessment, and health record management.

The paper "The Hierarchies of Multivalued Attribute Domains and Corresponding Applications in Data Mining" described the usage of association rules among attributes as common knowledge patterns in providing potential useful information such as mobile users' interests. The authors concentrated on processing relations between objects and attributes. To reduce the number of association rules for improving computational efficiency, they proposed a method based on concept lattice and attribute analysis and established the connection between the functional dependencies in original relations and their corresponding 'rough' relations. The experiments proved that the method was feasible and effective in the reduction of association rules.

The paper "Automatic Approach of Sentiment Lexicon Generation for Mobile Shopping Reviews" addressed the need of processing large amount of user comments on various products with the increasing use of smartphones. The sentiment analysis of the product reviews heavily relied on the quality of sentiment lexicons. Focusing on the generation of high quality sentiment lexicons, the authors proposed an automatic approach for constructing a domain-specific sentiment lexicon by considering the relationship between sentiment words and product features in the mobile shopping reviews. The generated lexicon was evaluated with a sentiment classification task using product reviews in both English and Chinese. The experiment showed the effectiveness of the proposed sentiment lexicon generation approach in the mobile environment.

The paper "Using Sentence-Level Neural Network Models for Multiple-Choice Reading Comprehension Tasks" studied the multiple-choice task for reading comprehension based on several test datasets. It assessed the usefulness of sentence comprehension versus word comprehension. The authors proposed a sentence-level neural network model using LSTM network and trained a sentence-level attention model to obtain sentence-level representation from sentence embedding in documents. The experiment showed that the model outperforms various state-of-the-art baselines on the multiple-choice reading comprehension task.

The paper "Recommending Mobile Microblog Users via a Tensor Factorization based on User Cluster Approach" explored the factor of user influence for microblog user recommendation in mobile social network. It highlighted the weakness of existing user influence analysis research for ignoring user temporal features and failing to filter marketing users with low influence. The authors proposed a tensor factorization-based user cluster model for influence analysis. This model identified latent influential users and constructed a feature tensor for user influence prediction and microblog user recommendation. The experiment showed the model was able to recognize latent influential users and improve existing recommendation systems through influence analysis.

\section{Conflicts of Interest}

The authors declare that there are no conflicts of interest regarding the publication of this article.

\section{Acknowledgments}

We would like to express our gratitude to all the authors for their high-quality submissions and all the reviewers for their timely and professional reviews.

\footnotetext{
Tianyong Hao

Raymond Wong Zhe He

Haoran Xie

Tak-Lam Wong

Fu Lee Wang
} 


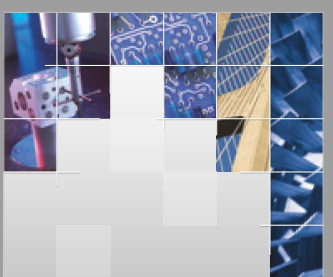

\section{Enfincering}
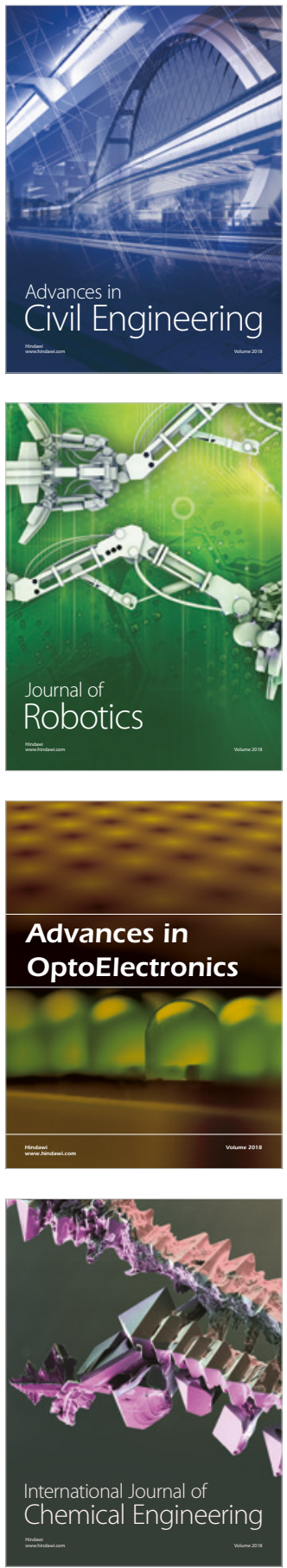

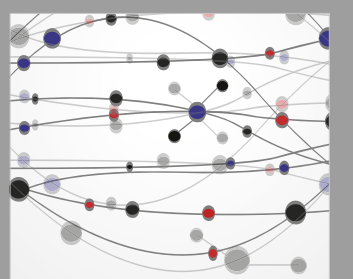

\section{Rotating \\ Machinery}

The Scientific World Journal

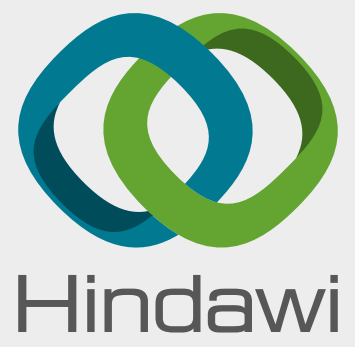

Submit your manuscripts at

www.hindawi.com
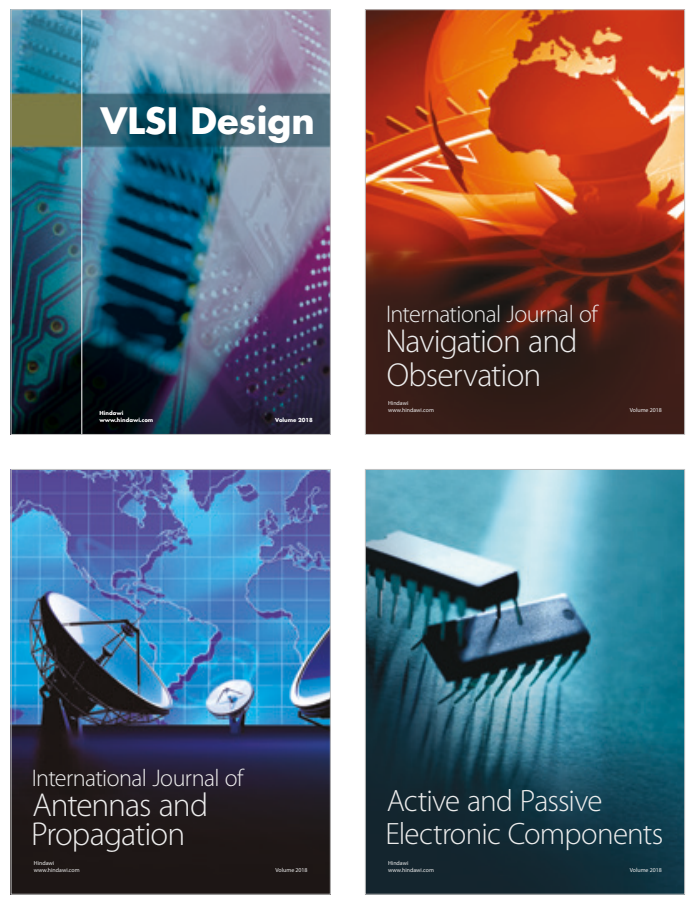
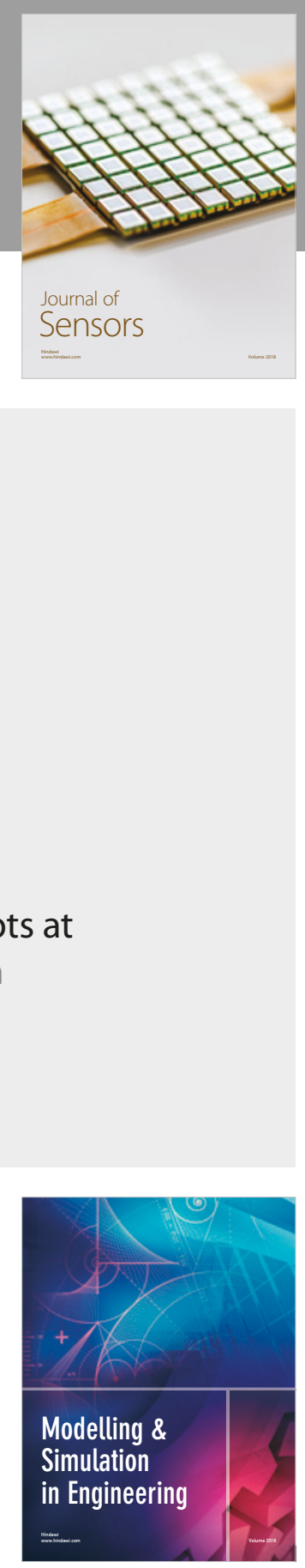

\section{Advances \\ Multimedia}
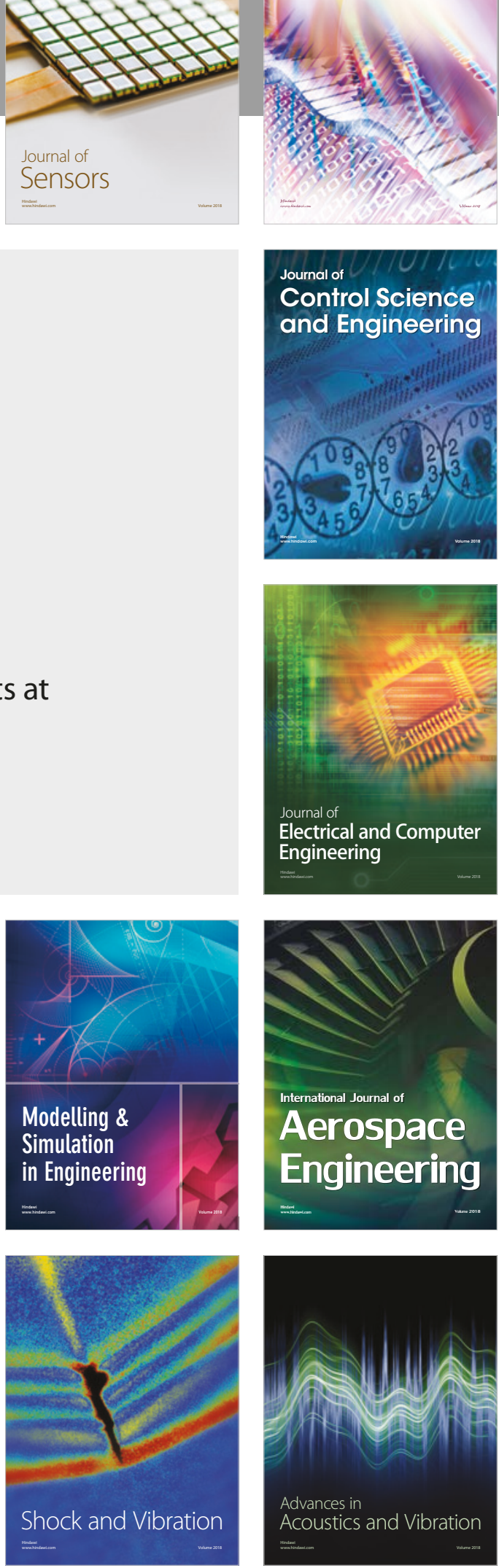